B. L. Osofsky

Nagoya Math. J.

Vol. 119 (1990), 107-114

\title{
INJECTIVE MODULES OVER TWISTED POLYNOMIAL RINGS
}

\author{
BARBARA L. OSOFSKY
}

Differential polynomial rings over a universal field and localized twisted polynomial rings over a separably closed field of non-zero characteristic twisted by the Frobenius endomorphism were the first domains not divisions rings that were shown to have every simple module injective (see [C] and [C-J]). By modifying the separably closed condition for the polynomial rings twisted by the Frobenius, the conditions of every simple being injective and only a single isomorphism class of simple modules were shown to be independent (see [O]). In this paper we continue the investigation of injective cyclic modules over twisted polynomial rings with coefficients in a commutative field.

Let $\kappa$ be a field and $\sigma$ an endomorphism of $\kappa$. We can then form the twisted polynomial ring $R=\kappa[X ; \sigma]$ with

$$
R=\left\{\sum_{i=0}^{n} \alpha_{i} X^{i} \mid n \in \mathbf{Z}, \alpha_{i} \in \kappa\right\}
$$

under usual polynomial addition and multiplication given by the relation

$$
X \alpha=\sigma(\alpha) X .
$$

We are interested in non-zero cyclic injective left modules over this ring $R$.

It is well known (see [J]) that $R$ is a left Euclidean domain using the degree function, and so a left principal ideal domain. Thus a left $R$-module is injective if and only if it is divisible (see [R, page 70]).

The field $\kappa$ is an $R$-module under the action

$$
\left(\sum_{i=0}^{n} p_{i} X^{i}\right) \cdot \alpha=\sum_{i=0}^{n} p_{i} \sigma^{i}(\alpha) .
$$

Using this action, we get

Theorem 1. Let $\kappa$ be a field and $R=\kappa[X ; \sigma]$. Then the following are equivalent:

Received September 9, 1989. 
(1) For every $q \in R$ with constant term $\neq 0, R / R q$ is injective.

(2) There exists a non-zero $\alpha \in \kappa$ with $R / R(X-\alpha)$ injective.

(3) For every $t \in \kappa$ and every non-zero $p=\sum_{i=0}^{l} p_{i} X^{i} \in R$, there is an $\alpha \in \kappa$ such that $p \cdot \alpha=t$, that is, the " $\sigma$-polynomial" equation $\sum_{i=0}^{n} p_{i} \sigma^{i}(\alpha)-$ $t=0$ has a root in $\kappa$.

(4) The right-left analog of any of the above conditions.

Proof. Clearly (1) $\Rightarrow(2)$.

We now examine injectivity of cyclic modules by looking at divisibility properties of quotients of $R$ in order to complete the proof.

It is easy to see that the twisting endomorphism must be an automorphism if a twisted polynomial ring has a non-zero cyclic injective module. In particular, let $q(X)=\sum_{i=0}^{k} q_{i} X^{i}$ be a monic polynomial of degree $k>0$. Then $X^{k} \equiv-\sum_{i=0}^{k-1} q_{i} X^{i}$ modulo $R q$, and $X p$ has constant term in $\sigma[\kappa] q_{0}$ modulo $R q_{0}$ for any polynomial $p$, so any $\alpha$ not in $\sigma[\kappa] q_{0}$ cannot be divisible by $X$ modulo $q$. Hence we will assume that $\sigma$ is onto.

We observe that

$$
\sum_{i=0}^{l} p_{i} X^{i}=\sum_{i=0}^{l} X^{i} \sigma^{-i}\left(p_{i}\right)
$$

Thus there is left-right symmetry and everything we say about left modules also holds on the right.

Now let $p=\sum_{i=0}^{l} p_{i} X^{i}$ and $q=\sum_{j=0}^{k} q_{j} X^{j}$ be two elements of $R$. The statement that $R / R q$ is divisible by $p$ means that for every $r \in R$ there is an $s \in R$ such that $r-p s \in R q$, that is, $R=p R+R q$. By the left Euclidean algorithm we can take $s$ of degree less than $q$. By the left and right Euclidean algorithms, to test this divisibility we need only show that every $r$ of degree less than $\min \{\operatorname{deg}(p)$, $\operatorname{deg}(q)\}$ lies in $p R+R q$. Let $\operatorname{deg}(p)=l$ and $\operatorname{deg}(q)=k$. We then have $R=p R+R q$ if and only if for all $\sum_{i=0}^{\min (k, l)-1} \tau_{i} X^{i}$,

$$
\left(\sum_{i=0}^{l} p_{i} X^{i}\right)\left(\sum_{j=1}^{k-1} \alpha_{j} X^{j}\right)+\left(\sum_{i=0}^{l-1} \beta_{i} X^{i}\right)\left(\sum_{j=0}^{k} q_{j} X^{j}\right)=\sum_{i=0}^{\min (k, l)-1} \tau_{i} X^{i}
$$

For convenience, we also set $\tau_{i}=0$ for $i>\min (k, l)-1$.

We thus get the systems (linear over $\kappa[X ; \sigma]$ ) of $k+l$ equations in $k+l$ variables

$$
\left(\sum_{i+j=n} p_{i} \sigma^{i}\left(\alpha_{j}\right)\right)+\left(\sum_{i+j=n} \beta_{i} \sigma^{i}\left(q_{j}\right)\right)=\tau_{n} \quad \text { for } 0 \leq n \leq k+l-1 .
$$


We abbreviate this system

(*)

$$
\mathbf{A} \cdot\left(\begin{array}{c}
\alpha_{0} \\
\alpha_{1} \\
\vdots \\
\alpha_{k-1} \\
\beta_{0} \\
\vdots \\
\beta_{l-1}
\end{array}\right)=\left(\begin{array}{c}
\tau_{0} \\
\tau_{1} \\
\vdots \\
\tau_{k-1} \\
\tau_{k} \\
\vdots \\
\tau_{k+l-1}
\end{array}\right)
$$

where $\mathbf{A}$ is the $(k+l) \times(k+l)$ matrix

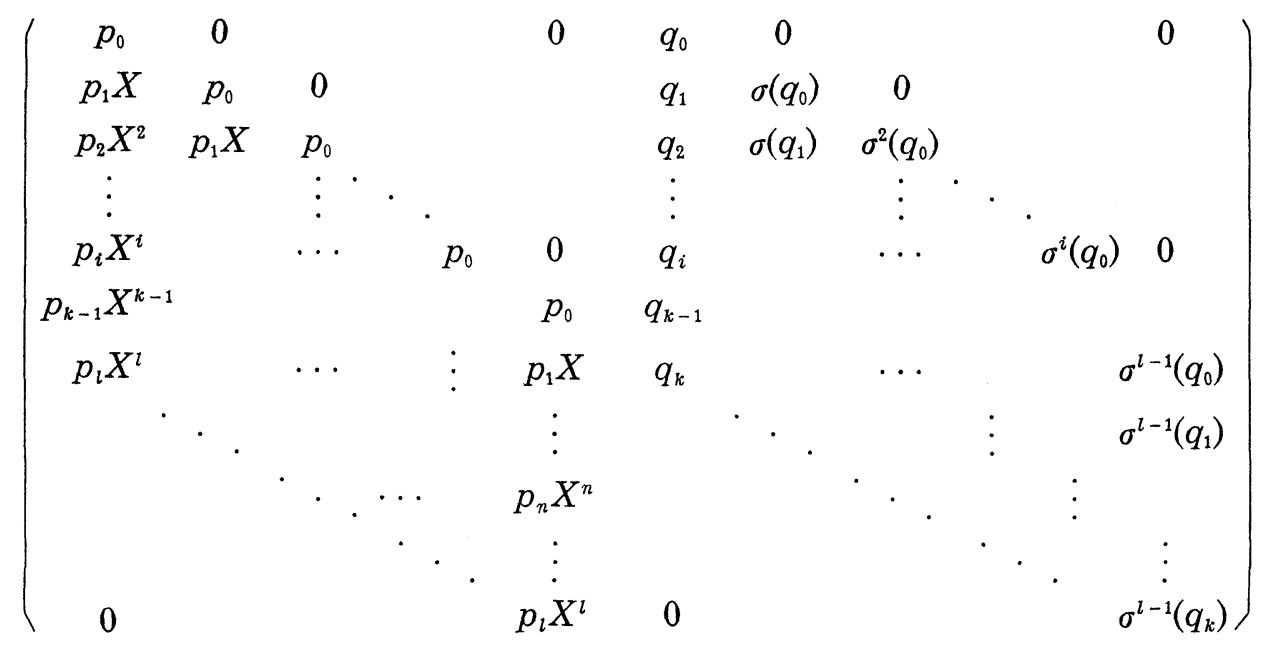

pictured here as though $k=l$. Modifications for $k \neq l$ are very minor.

The significant properties of $\mathbf{A}$ are that the first $k$ columns correspond to $p$ and the last $l$ columns correspond to $q$. The left $(k+l) \times k$ submatrix has the constant $p_{0}$ on its diagonal, zeros above the diagonal, and multiples of $X$ below the diagonal. The right $(k+l) \times l$ submatrix also has zeros above its diagonal and its bottom $l$ rows form an upper triangular submatrix with diagonal entries $\sigma^{i}\left(q_{k}\right)$. These entries $\sigma^{i}\left(q_{k}\right)$ are also on the diagonal of $\mathbf{A}$.

Let $\mathbf{A}_{1}$ denote the upper left $k \times k$ submatrix of $\mathbf{A}$.

Since $q$ has degree $k, \sigma^{i}\left(q_{k}\right) \neq 0$ for $0 \leq i \leq l-1$. Also,

$$
X R+R q=R \Longleftrightarrow R X+R q=R \Longleftrightarrow q_{0} \neq 0
$$

so we may take $p_{0} \neq 0$ and $q_{0} \neq 0$ in testing to see if $R / R q$ is injective. 
We now proceed using Gaussian elimination in a manner similar to that used in [O].

By pivoting successively on $\sigma^{l-1}\left(q_{k}\right), \sigma^{l-2}\left(q_{k}\right), \cdots, q_{k}$ we can make every non-diagonal entry in the last $l$ columns 0 (and the diagonal entries 1). In this process, all polynomials which are added to the entries in the upper left $k \times k$ submatrix $\mathbf{A}_{1}$ are multiples of $X$. Let $\boldsymbol{a}_{i}$ denote the $i$ th row of $\mathbf{A}_{1}$, and assume $\sum_{i=0}^{k} r_{i} a_{i}=0$. If some $r_{i} \neq 0$, there must be a $j$ with $r_{j}$ of smallest order (the smallest power of $X$ which occurs with non-zero coefficient). Then the $j$ th entry of $\sum_{i=0}^{k} r_{i} a_{i}$ contains a term of smallest order from $a_{j}$ which cannot be cancelled by any other term in $\sum_{i=0}^{k} r_{i} a_{i}$, a contradiction. By a series of elementary row operations using the Euclidean algorithm to decrease degree, we can bring $\mathbf{A}_{1}$ into lower echelon form, and the preceding discussion shows that we can never get a zero polynomial on the diagonal, as that would give us a zero row.

Doing the same row operations on the column of constants in (*) as were done in the matrix $\mathbf{A}$ gives us a new system

$$
\mathbf{L} \cdot\left(\begin{array}{c}
\alpha_{0} \\
\alpha_{1} \\
\vdots \\
\alpha_{k-1} \\
\beta_{0} \\
\vdots \\
\beta_{l-1}
\end{array}\right]=\left(\begin{array}{c}
\hat{\tau}_{0} \\
\hat{\tau}_{1} \\
\vdots \\
\hat{\tau}_{k-1} \\
\hat{\tau}_{k} \\
\vdots \\
\hat{\tau}_{k+l-1}
\end{array}\right)
$$

where $\mathbf{L}$ is a lower triangular matrix with non-zero polynomials on the diagonal and the $\hat{\tau}_{i}$ are obtained from the $\tau_{i}$ by multiplication by an invertible matrix. In summary, this system has a solution for any $\left\{\hat{\imath}_{i} \mid 0 \leq i \leq k+l-1\right\} \Leftrightarrow p R+R q=R$.

We note that the $R$-module $\kappa$ is isomorphic to $R / R(X-1)$. Statement (3) is precisely the statement that $\kappa$ is a divisible $R$-module. We can now complete the proof of the theorem.

Given (3), the equations (**) can be solved by forward substitution, so $(3) \Rightarrow(1)$.

For $(2) \Rightarrow(3)$, we take $k=1$ and $q=X-\alpha$ with $\alpha \neq 0$. Then 


$$
\mathbf{A}=\left(\begin{array}{ccccc}
p_{0} & \alpha & & & \\
p_{1} X & 1 & \sigma(\alpha) & & \\
p_{2} X^{2} & 0 & 1 & & \\
\vdots & & \ddots & \sigma^{l-1}(\alpha) \\
\vdots & & & \ddots & 1
\end{array}\right)
$$

and $\mathbf{L}$ has $(1,1)$ entry $\sum_{i=0}^{l}(-1)^{i} p_{i} \prod_{j=0}^{i-1} \sigma^{j}(\alpha) X^{i}$. If $\alpha \neq 0$ and $R / R(X-\alpha)$ is divisible by all non-zero polynomials $p$, then the coefficients of the $(1,1)$ entry of $\mathbf{L}$ are arbitrary, so every " $\sigma$-polynomial" equation must have a solution, and $(2) \Rightarrow(3)$.

Since $\kappa$ is commutative, (3) is left-right symmetric, so one gets (4) by this symmetry.

A module over a ring or object in an $\mathscr{A} \mathscr{B} 5$ category is called $C S$ (or extending, or having property $\mathrm{C} 1$, or ...) provided every submodule is essential in some direct summand. In [O-S], the condition that every cyclic $R$-module is $C S$ is studied as an example to illustrate the main result. That paper contains a sketch of a proof that every cyclic $R$-module is $C S$ implies that, for any simple $R$-module $M$ with injective hull $E(M)$, if the annihilators of non-zero elements of $M$ are not two-sided, then $E(M) / M$ is semi-simple. Theorem 1 enables us to complete the discussion of when every cyclic $R$-module is $C S$ begun in [O-S], filling in details just sketched there.

Lemma A. Let $\alpha \in \kappa$. Then $R / R(X-\alpha)$ is isomorphic to $R / R(X-1)$ $\Leftrightarrow \alpha=\sigma(\beta) / \beta$ for some $\beta \neq 0$ in $\kappa$.

Proof. $\quad R / R(X-1) \cong R / R(X-\alpha) \Leftrightarrow \exists \beta \in \kappa \backslash 0$ with $(X-1) \beta \in R(X-\alpha)$ $\Leftrightarrow \exists \beta \in \kappa \backslash 0$ with $\sigma(\beta) X-\beta \in R(X-\alpha) \Leftrightarrow \exists \beta \in \kappa \backslash 0$ with $\alpha=\beta / \sigma(\beta)$.

Lemma B. Let $U$ and $S$ be modules over some arbitrary ring $\mathscr{R}$ with 1. Assume $S=\mathscr{R}$ s is simple, and $U$ is a uniserial module with a unique composition series $U \supset U_{1} \supset U_{2} \supset 0$, with $S \cong U_{1} / U_{2}$. Then $M=U \oplus S$ is not CS.

Proof. Let $u+U_{2}$ map to $s$ in the isomorphism from $U_{1} / U_{2}$ to $S$, where $u \in U_{1}$. Since $U$ is uniserial, $\mathscr{R} u$ must have composition length 2, and the same is true for $\mathscr{R}(u+s)=N \subset M$. We observe that socle $(N)$ $=U_{2}$ and $N \oplus S$ is the only submodule of $M$ of length 3 containing $N$. Thus $N$ has no proper essential extensions in $M$. However, $N$ cannot be 
a direct summand of $M$ since $M / N$ is a direct sum of two simple modules whereas the socle of $M$ is $U_{2} \oplus S$ and $U_{2} \subset N$.

Lemma C. Let $\mathscr{R}$ be a principal left ideal domain, and let $p$ and $q$ generate maximal left ideals of $\mathscr{R}$. If $M$ is a simple $\mathscr{R}$-module not divisible by $p$ and $\mathscr{R} / \mathscr{R} p$ is not divisible by $q$, then $\mathscr{R}$ has a uniserial module $\mathscr{R} u \supset U_{1} \supset U_{2} \supset 0$ of composition length 3 with $U_{1} / U_{2} \cong \mathscr{R} / \mathscr{R} p$.

Proof. Let $E=E(M)$ denote an injective hull of $M$. Let $m \in M \backslash p M$. Then there is an $x \in E$ with $p x=m$. Since $\mathscr{R}$ is hereditary, $E / M$ is injective. Then $\mathscr{R} x /(\mathscr{R} x \cap M)$ has an injective hull $E^{\prime}$ in $E / M$. In $E$ there is an element $u \notin \mathscr{R} x$ with $u+M \in E^{\prime}$ and $\mathscr{R} q u+M=\mathscr{R} x+M$. Then $\mathscr{R} u \supset \mathscr{R} x \supset M \supset 0$, and one can easily check that $\mathscr{R} u$ has the required properties.

Theorem 2. Let $\kappa$ be a field and $R=\kappa[X ; \sigma]$. Then the following are equivalent:

(1) For every $q \in R, R / R q$ is $C S$.

(2) Either $\sigma$ is the identity or for every $q \in R$ with constant term $\neq 0$, $R / R q$ is injective.

Proof. $\quad(2) \Rightarrow(1)$ is reasonably elementary. The ring itself is a uniform module and so $C S$, and if $\sigma$ is the identity, other cyclics are $C S$ by the basis theorem for finitely generated Abelian groups. If $p \in R \backslash 0, p=$ $q X^{j}=X^{j} q^{\prime}$ for some $j \in \omega$ and $q, q^{\prime} \in R$ with constant term $\neq 0$. Since $R$ is a pid, $R=R X^{j}+R q^{\prime}$ and $R / R p$ has a natural map onto $R / R X^{j} \oplus$ $R / R q^{\prime}$. Computing $\kappa$-dimensions shows that this map is one to one. We observe that $R / R X^{j}$ is quasi-injective and $R / R q^{\prime}$ is injective and there are no non-zero homomorphisms between submodules of one and submodules of the other. Thus $R / R q$ is quasi-injective and so $C S$.

To show that $(1) \Rightarrow(2)$ we may assume that is $\sigma$ not the identity. Assume $R$ contains a $q^{\prime}$ with non-zero constant term such that $M=R / R q^{\prime}$ is not divisible by $X-1$. Then since $M$ is a finite dimensional vector space over $\kappa$, it is an $R$-module of finite length, and so has a simple composition factor which is not divisible by $p=X-1$. Thus we may assume that $M$ is simple. By Theorem $1, R / R(X-1)$ cannot be injective or $M$ would be, so $R / R(X-1)$ is not divisible by some non-zero irreducible polynomial $q$. By Lemma $\mathrm{C}$, there is an $s \in R$ with $R / R s \cong R u$ uniserial of length 3 with middle factor isomorphic to $R / R(X-1)$. Since 
$R / R s$ has only one maximal submodule, there is at most one $\alpha \in \kappa$ with $R(X-\alpha) \supset R s$. We are assuming that $\sigma$ is not the identity, so there is a $\beta \in \kappa$ with $\sigma(\beta) \neq \beta$. Then at least one $\gamma \in\{1, \beta / \sigma(\beta)\}$ satisfies $s \notin R(X-\gamma)$. By Lemma $\mathrm{A}, R / R(X-\gamma) \cong R / R(X-1)$. Then $R s+R(X-\gamma)=R$, so $R /(R s \cap R(X-\gamma)) \cong R / R s \oplus R / R(X-\gamma)$ is not $C S$ by Lemma B.

We conclude that every cyclic $C S$ implies that for all $q$ with constant term $\neq 0$ (and indeed for every non-zero $q$ ), $R / R q$ is divisible by $X-1$, that is, $(X-1) R+R q=R$. But that same equation may be interpreted as saying that the right $R$-module $R /(X-1) R$ is divisible by every nonzero $q \in R$, and so injective. By Theorem 1 , for every $q$ with constant term $\neq 0, R / R q$ is injective.

Remark. If every cyclic $R$-module is $C S$ and $\sigma$ is not the identity, then for every polynomial $q$ with non-zero constant term, $q R+R q=R$. In particular, $R$ cannot have any two-sided ideals other than $R, R X^{m}$, and 0 . It is well known that the two-sided ideals of $R$ are generated by powers of $X$ and by polynomials in $X^{n}$ with coefficients in the fixed field of $\sigma$, where $\sigma^{n}$ is the identity. Thus every cyclic $R$-module $C S$ and $\sigma$ of finite order imply that $\sigma$ is of order 1 , i.e. equal to the identity.

To get a feel for what " $\sigma$-polynomial” equations look like, it pays to look at some examples. First, let us assume that $\kappa$ is a perfect field of characteristic $p>0$ and $\sigma$ is the Frobenius map $\alpha \mapsto \alpha^{p}$. Then the equation

$$
\left(\sum_{i=0}^{n} q_{i} X^{i}\right) \cdot \alpha=\beta \quad \text { becomes } \quad \sum_{i=0}^{n} q_{i} \alpha^{p^{i}}=\beta
$$

which is an ordinary polynomial equation in $\alpha$. Note that polynomial is considerably different than the original polynomial in $R$. Among other things, it has ordinary derivative the constant $q_{0}$ so it is separable if $q_{0} \neq 0$, and its degree is a power of $p$. As observed in [O], all such ordinary polynomials may have roots in $\kappa$ without $\kappa$ being algebraically closed. If $\kappa$ is finite, then $\sigma$ is of finite order so by the above remark, some cyclic $R$-module, and hence the $R$-module $\kappa$, is not injective. In particular, the annihilator in $\kappa$ of $X-1$ is of order $p$, so the set of elements divisible by $X-1$ has order $|\kappa| / p$.

The above example may be somewhat misleading, since the operation of an element of $R$ on $\alpha$ gives a polynomial in $\alpha$. So let us now look at the case that $\kappa=\mathbf{C}$, the field of complex numbers, and $\sigma(z)=\bar{z}$, the 
complex conjugate of $z$. Then $\sigma$ is of order 2 , and $(X-1) \cdot \mathbf{C}=\mathbf{R} i$. If we wish to extend $\mathbf{C}$ to a field $\kappa_{1}$ in which every equation of the form $(X-1) \cdot \kappa_{1}=\beta$ has a root, adjoin a transcendental $\tau$ to $\mathbf{C}$ and extend complex conjugation to $\sigma:\left(\sum_{j=0}^{n} q_{j} \tau^{j}\right) \mapsto\left(\sum_{j=0}^{n} \bar{q}_{f}(\tau+1)^{j}\right)$. Clearly $\sigma$ is an automorphism of $\mathbf{C}[\tau]$ and so of $\kappa_{1}$. Then $\kappa_{1}$ is an $R$-R bimodule, and $(X-1) \cdot \tau=1$ so $(X-1) \cdot \kappa_{1} \supseteq \mathbf{R}$. Computations show that applying $(X-1)$. to higher powers of $\tau$ and $i$ times those powers alternately gives real and imaginary parts of coefficients of every power of $\tau$, so one gets that $\kappa_{1}$ is divisible by $X-1$. It is not, however, divisible by $X-\tau$. If $q=\sum_{i=0}^{n} q_{i} X^{i} \in R$ has $q_{0} q_{n} \neq 0$, and $q \cdot \alpha=\beta$ has no solution in $\kappa_{1}$, we may force it to have a solution in an extension of $\kappa_{1}$ by adjoining new transcendentals $\left\{x_{0}, \cdots, x_{n-1}\right\}$ to $\kappa_{1}$ and extending $\sigma$ to this new $\kappa_{2}$ by $\sigma: x_{i} \mapsto x_{i+1}$ for $0 \leq i \leq n-2$ and $\sigma: x_{n-1} \mapsto\left(\beta-\sum_{i=0}^{n-1} q_{i} x_{i}\right) / q_{n}$. Iterating this procedure carefully will enable us to get a " $\sigma$-algebraic closure" of the original field for which the new field is injective over the new $R$. It will look nothing like the algebraically closed field $\mathbf{C}$.

We conclude with an obvious conjecture, namely, if some $R / R_{q}$ is injective with $q$ having non-zero constant term, then so is $R / R(X-1)$. A computational proof seems very difficult, as not all polynomials appear on the diagonal of the lower triangular $\mathbf{L}$ in equation (**).

\section{REFERENCES}

[C] J. H. Cozzens, Homological properties of the ring of differential polynomials, Bull. Amer. Math. Soc., 76 (1970), 75-79.

[C-J] J. H. Cozzens and J. L. Johnson, An application of differential algebra to ring theory, Proc. Amer. Math. Soc., 31 (1972), 354-356.

[J] N. Jacobson, "The theory of rings," American Mathematical Soc., Providence, 1943.

[0] B. L. Osofsky, On twisted polynomial rings, J. Algebra, 4 (1971), 597-607.

[O-S] B. L. Osofsky and P. F. Smith, Cyclic modules whose quotients have complements direct summands, J. Algebra, to appear.

[R] J. J. Rotman, "An introduction to homological algebra," Academic Press, Inc., Orlando San Diego, 1979.

Department of Mathematics

Rutgers University

New Brunswick, NJ 08903

U.S.A. 